\title{
Sex ratio and Wolbachia infection in the ant Formica exsecta
}

\author{
LAURENT KELLER*†, CATHY LIAUTARD†, MAX REUTER†, WILLIAM D. BROWN† \\ LOTTA SUNDSTRÖM $† \S \&$ MICHEL CHAPUISAT† \\ $\dagger$ Institute of Ecology, University of Lausanne, Bâtiment de Biologie, 1015 Lausanne, Switzerland, $\$$ Department of \\ Biology, Syracuse University, 108 College Place, Syracuse, NY 13244-1270, U.S.A. and §Department of Ecology and \\ Systematics, Division of Population Biology, PO Box 17, FIN-00014 Helsinki, Finland
}

\begin{abstract}
Sex allocation data in social Hymenoptera provide some of the best tests of kin selection, parentoffspring conflict and sex ratio theories. However, these studies critically depend on controlling for confounding ecological factors and on identifying all parties that potentially manipulate colony sex ratio. It has been suggested that maternally inherited parasites may influence sex allocation in social Hymenoptera. If the parasites can influence sex allocation, infected colonies are predicted to invest more resources in females than non-infected colonies, because the parasites are transmitted through females but not males. Prime candidates for such sex ratio manipulation are Wolbachia, because these cytoplasmically transmitted bacteria have been shown to affect the sex ratio of host arthropods by cytoplasmic incompatibility, parthenogenesis, male-killing and feminization. In this study, we tested whether Wolbachia infection is associated with colony sex ratio in two populations of the ant Formica exsecta that have been the subject of extensive sex ratio studies. In these populations colonies specialize in the production of one sex or the other. We found that almost all F. exsecta colonies in both populations are infected with Wolbachia. However, in neither population did we find a significant association in the predicted direction between the prevalence of Wolbachia and colony sex ratio. In particular, colonies with a higher proportion of infected workers did not produce more females. Hence, we conclude that Wolbachia does not seem to alter the sex ratio of its hosts as a means to increase transmission rate in these two populations of ants.
\end{abstract}

Keywords: Formica exsecta, host manipulation, sex ratio, virulence, Wolbachia.

\section{Introduction}

Social Hymenoptera have been key organisms for the study of sex ratio theory and kin selection (Bourke \& Franks, 1995; Crozier \& Pamilo, 1996; Bourke, 1997; Queller \& Strassmann, 1998; Chapuisat \& Keller, 1999; Keller \& Reeve, 1999). The haplodiploid sex determination system (with males arising from unfertilized eggs and females from fertilized eggs) results in asymmetries of genetic relatedness among colony members and a parent-offspring conflict over relative allocation of resources to the two sexes (Trivers \& Hare, 1976; Nonacs, 1986; Bourke \& Franks, 1995; Crozier \& Pamilo, 1996). Because queens are equally related to both sexes of reproductives reared in their colony, their fitness is maximized by an even sex ratio investment. In contrast, workers are more related to their sisters than

*Correspondence. E-mail: Laurent.keller@ie-zea.unil.ch to their brothers, and their inclusive fitness is maximized by an increased investment in female brood.

Worker control of sex allocation not only leads to a female-biased population sex ratio, it can also cause variation in sex allocation between colonies with different kin-structure. Split sex ratio theory (Boomsma \& Grafen, 1990) predicts that workers in colonies with a relatedness asymmetry (RA, here defined as the higher relatedness of workers to females than males in their colony) above the population average should mainly specialize in the production of females. In contrast, workers in colonies with a RA below the average should favour the production of mostly males. Several studies have confirmed this prediction (Queller \& Strassmann, 1998). For example, in single-queen colonies of Formica truncorum and $F$. exsecta, nests headed by singly mated queens produce mostly females, whereas nests headed by multiply mated queens specialize on male production (Sundström, 1994; Sundström et al., 1996). However, in both species a large part of the variance in sex allocation 
is not explained by variation in relatedness asymmetry. Furthermore, in a population of $F$. exsecta with multiple queens per nest and in several other species, split sex ratios occurs but colony sex ratios are not correlated with relatedness asymmetry (Chapuisat \& Keller, 1999; Brown \& Keller, 2000; Helms, 1999). This suggests that other factors also influence colony sex allocation. One of the potential factors accounting for variations in colony sex ratio is the amount and type of resources available, possibly because it affects the relative cost of production of males and females (Rosenheim et al., 1996). In some species, variations in resource supply apparently affect sex ratio specialization (e.g. Deslippe \& Savolainen, 1995; Brown et al., 2001).

Recently, it has also been suggested that maternally inherited bacteria may have an influence on sex allocation in social Hymenoptera (Crozier \& Pamilo, 1993; Bourke \& Franks, 1995; Crozier \& Pamilo, 1996; Wenseleers \& Billen, 2000). Wolbachia, a group of intracellular bacteria related to Rickettsiae, stand as one of the most likely candidate organisms that may affect sex ratios. These bacteria are known to infect Hymenoptera and other arthropods, particularly their reproductive tissues (Rousset et al., 1992; Werren, 1997). They are transmitted through the egg cytoplasm and alter reproduction in their host in various ways. In particular, they are associated with induction of parthenogenesis, cytoplasmic incompatibility, feminization of genetic males and male killing. Because Wolbachia are transmitted through females but not males, they would benefit by manipulating their host colony to produce only female sexuals and no males because males are a reproductive dead end for the bacteria. If Wolbachia can bias colony sex ratios but not all colonies are infected, split sex ratio could arise. Colonies bearing Wolbachia would produce only or predominantly females, whereas uninfected colonies would specialize in males, to reduce the sex ratio bias at the population level.

The aim of this study is to determine whether Wolbachia infection is associated with variation in colony sex ratios in the ant Formica exsecta. This species has been extensively studied with regard to sex allocation and a preliminary study indicated that it harbours Wolbachia. Split sex ratios have been reported from two different populations that differ in their social organization.

In a Finnish population where colonies contain a single queen (monogyny), the population sex ratio investment was female biased; most colonies headed by a singly mated queen specialized in female production, whereas colonies headed by a multiply mated queen specialized in the production of males (Sundström et al., 1996). These results are consistent with the idea that colony sex ratios are under the control of workers.
By contrast, it seems unlikely that Wolbachia could be responsible for the association between queen mating frequency and colony sex ratios, because there is no obvious reason why singly mated queens should be more likely to be infected by Wolbachia.

The other study population, which is located in Switzerland, contains colonies which typically contain numerous queens (polygyny). In contrast to the Finnish population, no association between relatedness asymmetry and sex allocation was detected in the Swiss population. In that population sex ratio was highly male biased and only a minority of nests $(11 \%)$ produced females (Brown \& Keller, 2000; Brown \& Keller submitted).

We used a PCR assay to determine the prevalence of Wolbachia in workers from colonies specializing in male and female production in both the Swiss and Finnish populations. We used the same colonies that had been used in previous sex ratio studies to determine whether previous conclusions on factors affecting sex ratio might have been confounded by the presence of Wolbachia.

\section{Materials and methods}

\section{Colony sampling}

Our study included individuals from $71 F$. exsecta colonies. All these colonies had been part of earlier genetic and sex ratio studies (Sundström et al., 1996; Chapuisat et al., 1997b; Brown \& Keller, 2000).

We analysed 40 nests in a Swiss polygynous population. Half of the colonies were male producing and the other half were female producing, following Brown \& Keller's (2000) criteria. All samples analysed were adult workers. We analysed 31 nests from a Finnish monogynous population. Colony sex ratios were obtained from Sundström et al. (1996). We classified colonies as male producing if they produced more than $75 \%$ males and as female producing if they produced more than $63 \%$ queens. For our study, we chose 17 female-producing and 14 male-producing nests. The age of the workers analysed varied between colonies. DNA had been extracted from adult workers in 14 of these colonies and from worker pupae for the other 17 colonies. There was no significant association between the type of material analysed and colony sex ratio (Fisher's exact test, $P=0.07$ ).

\section{Wolbachia detection}

We tested for the presence of Wolbachia in five workers from each of the selected colonies. Wolbachia infection was detected by PCR-amplifying a fragment of the cell cycle gene Fts $Z$ using Wolbachia-specific primers. 
Primer sequences and PCR conditions are given in Wenseleers et al. (1998). A previous analysis (unpublished) showed that only the strain A was present in the Swiss population of $F$. exsecta. Thus, PCRs for this study were performed with primers specific for the strain A. PCR products were run on $2 \%$ agarose gels and visualized by ethidium bromide staining. Each sample was analysed twice and individuals were considered infected when positive for at least one of the PCRs.

Wolbachia infection was detected by PCR-amplifying a fragment of the cell cycle gene FtsZ, using Wolbachiaspecific primers.

We took several precautions to avoid artefacts in the Wolbachia screening. For each PCR, we used a positive as well as a negative control. The positive control was an individual known to carry Wolbachia. In the negative control, DNA was replaced by deionized water. Further, to avoid any bias of our results due to PCR conditions and/or loading effect, we alternated female- and maleproducing nests in PCRs and migrations on agarose gel. Thus, in each PCR and on each gel, half of the individuals were from a female-producing and the other half from a male-producing nest. Finally, we could exclude false negatives due to problems during DNA extraction, because all samples had previously been part of a microsatellite analysis and were known to contain DNA.

\section{Statistical analysis}

Because our two samples differed with respect to locality, social organization, and the type of material used, we performed separate statistical analysis on the data from Switzerland and Finland. We analysed the data using a generalized linear model (GLM) with a binomially distributed error and a logistic link function. Presence or absence of infection in individual workers was the dependent variable. For analysing the Swiss data, we entered the colony sex ratio (male-producing or female-producing) and colony nested within sex ratio as independent variables. For the Finnish data, we entered colony sex ratio, the material used (adult workers or pupae), the interaction of these two variables, as well as colony nested within sex ratio and material. The significance of factors was tested with Chi-squared tests. All analyses were performed with s-PLus 2000 (MathSoft Inc.).

\section{Results}

Our PCR method for detecting Wolbachia was reasonably reliable. In the Finnish population, 95 individuals $(61 \%)$ were scored as infected for the two PCRs, 11 $(7 \%)$ as infected once and $49(32 \%)$ as never infected.
Similar values were found in the Swiss population, with $96(47 \%), 25(13 \%)$ and $79(40 \%)$ individuals being scored as being infected twice, once and never, respectively. Overall, repeated PCR amplifications gave the same result for $120(87 \%)$ of the individuals analysed. The repeatability $r$ of the PCR results, measured as the proportion of between-individual variance among the total variance of PCR results (Falconer \& MacKay, 1996) was $r=0.86$ and $r=0.77$ for the Swiss and the Finnish populations, respectively.

Most of the colonies contained some infected workers (Fig. 1). In only four Swiss polygynous nests (10.0\%) and one Finnish monogynous nest $(3.2 \%)$, was no infected worker detected. In both populations there was no significant association between sex ratio and whether colonies contained infected workers (Switzerland, Fisher exact test, $P>0.9$; Finland, Fisher exact test, $P>0.9$ ). The proportion of infected colonies also did not differ significantly between the two populations (Fisher exact test, $P=0.38$ ). The overall percentage of colonies without infected workers was $7.0 \%$. The percentage of individuals infected was similar in the two populations. Sixty and $69 \%$ of the workers were scored as infected in the Swiss and Finnish populations, respectively.

Results from the GLM analyses are presented in Table 1 . The analyses revealed that the factor that had the greatest influence on the proportion of infected workers was the colony from which workers were sampled. The effect of colony was significant in both populations and explained as much as 58 and $39 \%$ of the variance in the Swiss and Finnish populations, respectively.

Overall, there was no clear association between sex ratio and the proportion of infected workers (Table 1). In the Swiss population, sex ratio was significantly associated with the proportion of infected workers but sex ratio explained only $2.6 \%$ of the total variance in the proportion of infected workers. Interestingly, the association was opposite to theoretical expectations if Wolbachia manipulated their host to increase their transmission rate. Female-producing colonies tended to have a lower number of infected workers $(2.6 \pm 2.0$ / 1.5 ; mean $\pm \mathrm{SD} /$ median) than male-producing colonies $(3.5 \pm 1.9 / 4.5)$. In the Finnish population, there was no significant association between these two variables and the number of infected workers was quite similar in female-producing $(3.5 \pm 1.7 / 4.0)$ and male-producing (3.4 $\pm 1.6 / 4.0)$ colonies (Fig. 2).

In the Finnish population, there was a significant association between the proportion of infected workers and the type of material analysed (Table 1). The proportion of infected individual was higher for pupae than workers, both in female- and male-producing 
Female-Producing

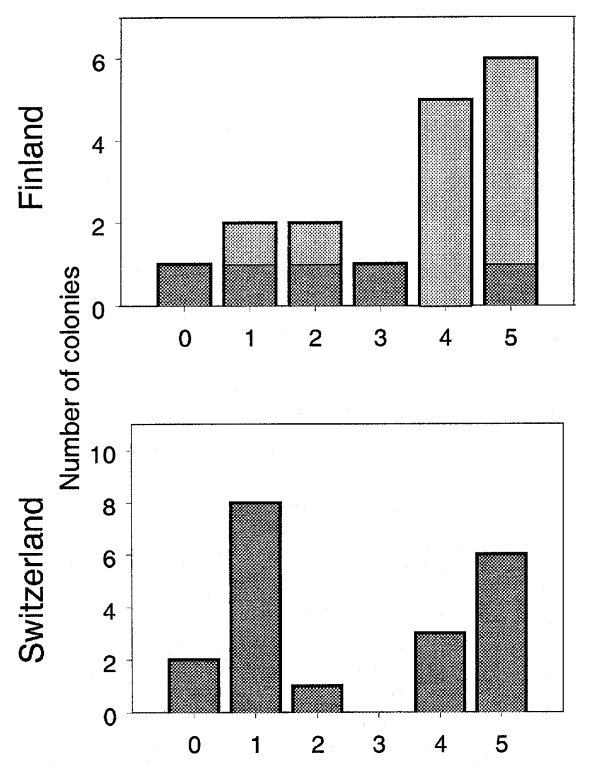

Male-Producing
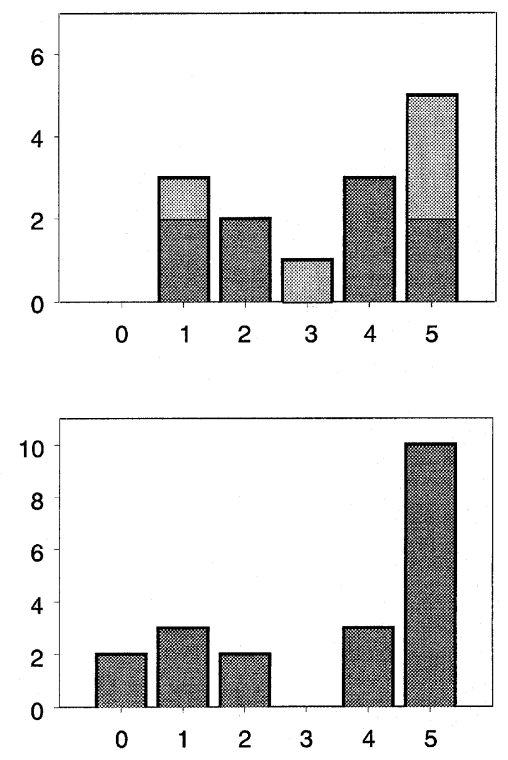

Fig. 1 Number of infected workers in male- and female-producing colonies in the Swiss and the Finnish populations of Formica exsecta. The $x$-axis gives the number of infected workers per colony (out of the five analysed). The $y$-axis gives the number of colonies with a given number of infected workers. For the Finnish population, data on worker pupae are given in light grey, and those on adult workers in black. In the Swiss population all individuals analysed were adult workers.

Table 1 Results from the GLM analyses for the Finnish population (a) and the Swiss population (b) of Formica exsecta. The dependent variable in both analyses was the infection status of workers (infected/uninfected). The table shows the independent variables entered in the analysis (Factor), the number of their degrees of freedom (d.f.), and their significance calculated by a Chi-squared test. Further, the percentage of the total variance explained by the significant factors is given (Explained V)

\begin{tabular}{lrcc}
\hline Factor & d.f. & $P$ & Explained V \\
\hline (a) Finland & & & \\
$\quad$ Sex ratio & 1 & $>0.5$ & - \\
$\quad$ Material & 1 & 0.0014 & $5.2 \%$ \\
$\quad$ Sex ratio $\times$ Material & 1 & 0.2 & - \\
$\quad$ Colony in & 27 & $<0.0001$ & $39.3 \%$ \\
$\quad$ (sex ratio + material) & & & \\
(b) Switzerland & & & \\
$\quad$ Sex ratio & 1 & 0.0088 & $2.6 \%$ \\
$\quad$ Colony in sex ratio & 38 & $<0.0001$ & $58.1 \%$ \\
\hline
\end{tabular}

colonies (Fig. 2). Overall, the type of material explained $5.2 \%$ of the total variance in the proportion of infected workers in Finland.

\section{Discussion}

Our data indicate that, in both the Swiss polygynous and Finnish monogynous populations, almost all colonies are infected with Wolbachia. Overall, only $7 \%$ of

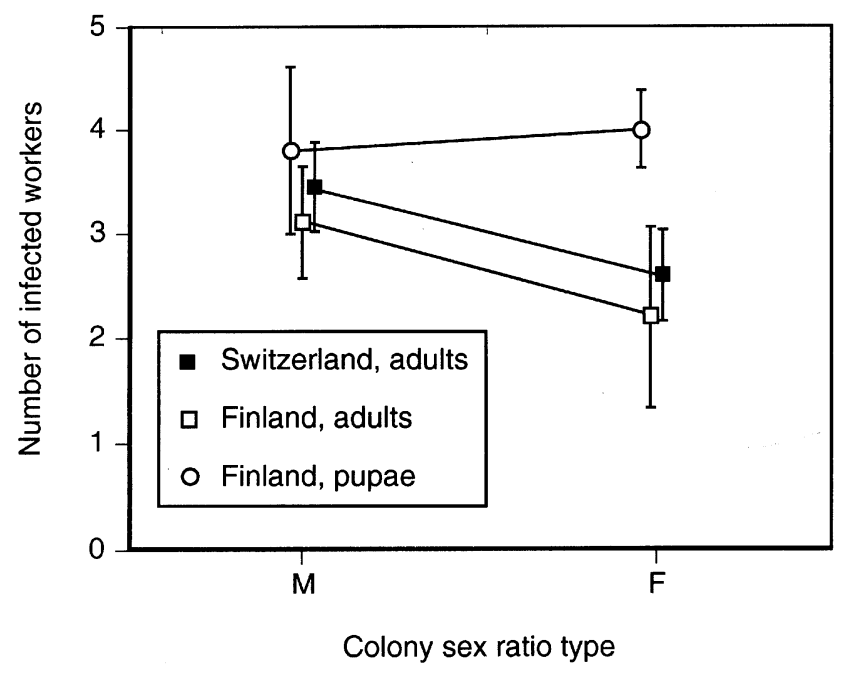

Fig. 2 Mean $( \pm \mathrm{SE})$ number of infected workers (out of the five analysed) in male- and female-producing colonies (M, F) of Formica exsecta. Data are given separately for adult workers in the Swiss population (black circles), and for adult workers (white circles) and worker pupae (squares) in the Finnish population.

the colonies contained no infected workers. In the Finnish monogynous population, although only five individuals were analysed per colony, only one of the 31 colonies had no infected workers, indicating that the large majority or even all the reproductive queens are infected. In the Swiss population it is more difficult to determine the proportion of infected queens because 
colonies can contain large number of queens and it is impossible to accurately assign maternity of workers in such colonies. However, the finding that the average proportion of infected workers is similar in both populations is suggestive that the proportion of infected reproductive queens might be similar in both populations, with perhaps all queens being infected.

The very high proportion of infected queens suggests that Wolbachia have efficient means to spread through the population. One very efficient mechanism favouring the spread of Wolbachia is cytoplasmic incompatibility, which occurs when an uninfected female mates with an infected male (Breeuwer \& Werren, 1990; Hoffmann et al., 1997). Such matings generally produce few or no female progeny as the result of abortive karyogamy (Lassy \& Karr, 1996). Because the other possible types of matings yield normal progeny numbers, the outcome of cytoplasmic incompatibility is lower fitness of uninfected females compared to infected females. This reproductive advantage of infected females results in the spread of Wolbachia through a population (Caspari \& Watson, 1959; Turelli, 1994). Interestingly, the spread of Wolbachia through cytoplasmic incompatibility should be even quicker in social Hymenoptera than in solitary species because uninfected females mated with infected males should produce few or no female workers (Vavre et al., 2000), and hence are unlikely to be successful in founding a new colony. More studies are needed to test whether the high prevalence of Wolbachia in $F$. exsecta is indeed due to the effect of cytoplasmic incompatibility.

In the Swiss population the prevalence of Wolbachia was slightly higher in male- than female-producing colonies. By contrast, there was no significant association between these two factors in the Finnish population. It is important to note that the association between colony type and prevalence of Wolbachia in the Swiss population was weak (sex ratio explained only $2.6 \%$ of the variance). Moreover, the association was opposite to what one would predict if Wolbachia altered colony sex ratios so as to increase their transmission rate. Thus, it seems likely that the weak but significant association between colony type and prevalence of Wolbachia is not due to an active manipulation of colony sex ratio by the parasite but rather results from the effect of a confounding ecological variable that affects both sex allocation and Wolbachia infection.

That ecological variables or intrinsic factors can affect the proportion of infected individuals is suggested by the higher proportion of infected worker pupae than worker adults in Finland. The higher prevalence of Wolbachia in pupae suggests that some workers lose infection with age or that the bacterial density decreases to levels undetectable with standard PCR methods, a result that may partially be explained by differences in the amount of fresh tissue between adults and pupae. A lower proportion of infected adult workers compared to worker pupae has also been found in another ant, Formica truncorum (T. Wenseleers, L. Sundström, and J. Billen, unpublished). The reasons underlying decreased proportion of infected workers with age are unknown. One possible explanation is that endoparasites are not selected to proliferate in workers that will never produce any female offspring (selection would even be negative if infection decreases worker efficiency, and thus overall colony productivity). Alternatively, the proportion of infected workers may decrease as a result of the possible exposure of adult workers to higher temperature. Increased temperature has been shown to decrease the density of Wolbachia in Drosophila bifasciata (Hurst et al., 2000). Thus, adult workers exposed to higher temperature while foraging may experience a decrease in the density of Wolbachia. It is also possible that differences in temperature (or other ecological variables) influence resource availability and thereby colony sex ratios, which might explain the weak association between these two variables in the Swiss population. Alternatively, female-producing colonies might be comprised of older workers, which would also account for an association between the proportion of infected workers and colony sex ratios.

The finding of no clear association between prevalence of Wolbachia and colony sex ratios together with the fact that all or almost all queens are infected indicate that sex ratio specialization in $F$. exsecta colonies cannot simply be accounted for by the presence or absence of Wolbachia. In particular, the finding that colonies with more infected workers tend to produce males in the Swiss populations indicates that Wolbachia do not manipulate their host into producing mostly or only females. Moreover, Wolbachia was as prevalent in the Swiss population, which has a male-biased population sex ratio, as in the Finnish population which shows a female-biased sex ratio.

There are several possible explanations for the apparent lack of sex ratio manipulation by Wolbachia in F. exsecta. One is proximate constraints arising from the mechanism of sex determination in ants (Wenseleers $\&$ Billen, 2000). In most ants, sex is probably determined by heterozygosity at one or several sex-determination loci with heterozygous individuals developing into females and homozygous and hemizygous individuals developing into males (Cook \& Crozier, 1995). Because Wolbachia-induced parthenogenesis generally relies on a cytogenetic mechanism of gamete duplication, yielding completely homozygous diploid offspring (Stouthamer \& Kazmer, 1994) this would lead to the production of 
diploid males which are generally sterile. An alternative mechanism by which Wolbachia might alter the sex ratio is manipulation of the proportion of viable fertilized eggs. This could be achieved if Wolbachia could decrease viability of haploid eggs or influence the mechanism of egg fertilization. In Nasonia vitripennis a maternal sex ratio distorter apparently induces a higher than normal frequency of egg fertilization (Skinner, 1982). However, such an effect has not yet been reported for Wolbachia (in ants a possible mechanism could be by acting on the muscles regulating transfer of sperm from the queen's spermatheca to the eggs). Additional studies in other ants would be helpful to ascertain whether Wolbachia generally has no effect on ant sex ratios. Studies of populations where Wolbachia has not yet spread to fixation would be particularly interesting as Wolbachia might have a greater effect on sex ratio variation between colonies in such a non-equilibrium situation.

\section{Acknowledgements}

We thank Nadège Duvoisin for assistance in the laboratory, Andrew Bourke, Dewayne Shoemaker, Tom Wenseleers and an anonymous referee for useful comments on the manuscript, and the Tvärminne Zoological Station for providing research facilities. This work was supported by several grants from the Swiss National Science Foundation, the 'Foundation du 450e' of the University of Lausanne, the Academy of Finland, the Swiss Office of Science and Technology in association with an EU research network financed via the Human Potential Programme.

\section{References}

BOOMSMA, J. J. AND GRAFEN, A. 1990. Intraspecific variation in ant sex ratios and the Trivers-Hare hypothesis. Evolution, 44, 1026-1034.

BOURKE, A. F. G. 1997. Sociality and kin selection in insects. In: Krebs, J. R. and Davies, N. B. (eds) Behavioural Ecology. An Evolutionary Approach, 4th edn, pp. 203-227. Blackwell, Oxford.

BOURKe, A. F. G. AND FRANKS, N. R. 1995. Social Evolution in Ants. Princeton University Press, Princeton.

BREeUwer, J. A. J. AND WERREN, J. H. 1990. Cytoplasmic incompatibility and bacterial density in Nasonia vitripennis. Genetics, 135, 565-574.

BROWN, W. D. AND KELLER, L. 2000. Colony sex ratios vary with queen number but not relatedness asymmetry in the ant Formica exsecta. Proc. R. Soc. B, 267, 1751-1757.

BRown, W. D., SUNDSTRÖM, L. AND KELLER, L. (2001) Sex allocation in wood ants: the roles of green replenishment, resource abundance and local resource competition. Ecology, in press.
CASPARI, E. AND WATSON, G. S. 1959. On the evolutionary importance of cytoplasmic sterility in mosquitoes. Evolution, 13, 568-570.

CHAPUiSAT, M. AND KELLER, L. 1999. Testing kin selection with sex allocation data in eusocial Hymenoptera. Heredity, 82, 473-478.

CHAPUISAT, M., GOUDET, J. AND KELlER, L. 1997a. Microsatellites reveal high population viscosity and limited dispersal in the ant Formica paralugubris. Evolution, 51, 475-482.

CHAPUiSAT, M., SUNDSTRÖM, L. AND KELLER, L. 1997b. Sex-ratio regulation - the economics of fratricide in ants. Proc. $R$. Soc. B, 264, 1255-1260.

COOK, J. AND CROZIER, R. H. 1995. Sex determination and population biology in the Hymenoptera. Trends Ecol. Evol., 10, 281-286.

CROZIER, R. H. AND PAMILO, P. 1993. Sex allocation in social insects: problems in prediction and estimation. In: Wrensch, D. L. and Ebberts, M. (eds) Evolution and Diversity of Sex Ratio in Insects and Mites, pp. 369-383. Chapman \& Hall, New York.

CROZIER, R. H. AND PAMILO, P. 1996. Evolution of social insect colonies. Sex Allocation and Kin-Selection. Oxford University Press, Oxford.

DESLIPPE, R. J. AND SAVOlainen, R. 1995. Sex investment in a social insect - the proximate role of food. Ecology, 76, 375-382.

FALCONER, D. S. AND MACKAY, T. F. C. 1996. Introduction to Quantitative Genetics, 4th edn. Longman, Essex.

HELMs, K. R. 1999. Colony sex ratios, conflict between queens and workers, and apparent queen control in the ant Pheidole desertorum. Evolution, 53, 1470-1478.

hofFMANN, A. A., TURELli, M. AND hARShMAN, L. G. 1997. Cytoplasmic incompatibility in insects. In: O'Neill, S. L., Hoffmann, A. A. and Werren, J. H. (eds) Influential Passengers; Inherited Microorganisms and Arthropod Reproduction, pp. 42-80. Oxford University Press, New York.

HURST, G. D., JOHNSON, A. P., SCHOLENBURG, J. H. AND FUYAMA Y. 2000. Male-killing Wolbachia in Drosophila: a temperature-sensitive trait with a threshold bacterial density. Genetics, 156, 699-709.

KELLER, L. AND REEVE, H. K. 1999. Dynamics of conflicts within insect societies. In: Keller, L. (ed.) Levels of Selection in Evolution, pp. 153-175. Princeton University Press, Princeton.

LASSY, C. W. AND KARR, T. L. 1996. Cytological analysis of fertilization and embryonic development in incompatible crosses of Drosophila simulans. Mechanisms Dev., 57, 47-58.

NONACS, P. 1986. Ant reproductive strategies and sex allocation theory. Quart. Rev. Biol., 61, 1-21.

QUELLER, D. C. AND STRASSMANN, J. E. 1998. Kin selection and social insects. BioScience, 48, 165-175.

RosenheIM, J. A., NONACS, P. AND MANGEL, M. 1996. Sex ratios and multifaceted parental investment. Am. Nat., 148, 501-535.

RousSet, F., Bouchon, D., PINTUREAU, B., JUChaUlt, P. ET AL. 1992. Wolbachia endosymbionts responsible for various alterations of sexuality in arthropods. Proc. R. Soc. B, 250, 91-98. 
STOUTHAMER, R. AND KAZMER, D. 1994. Cytogenetics of microbe-associated parthenogenesis and its consequences for gene flow in Trichogramma wasps. Heredity, 73, $317-327$.

SUNDSTRÖM, L. 1994. Sex ratio bias, relatedness asymmetry and queen mating frequency in ants. Nature, 367, 266-268.

SUNDSTRÖM, L., CHAPUISAT, M. AND KELlER, L. 1996. Conditional manipulation of sex ratios by ant workers - a test of kin selection theory. Science, 274, 993-995.

TRIVERS, R. L. AND HARE, H. 1976. Haplodiploidy and the evolution of the social insects. Science, 191, 249-263.

TURELLI, M. 1994. Evolution of incompatibility-inducing microbes and their hosts. Evolution, 48, 1500-1513.
VAVRe, F., FLeURY, F., VARALDI, J., FOUILLET, P. ET AL. 2000. Evidence for female mortality in Wolbachia-mediated cytoplasmic incompatibility in haplodiploid insects: Epidemiological and evolutionary consequences. Evolution, 54, 191-200.

WENSELEERS, T. AND BILlEN, J. 2000. No evidence for Wolbachia-induced parthenogenesis in the social Hymenoptera. J. Evol. Biol., 13, 277-280.

WENSELEERS, T., ITO, F., VAN BORM, S., HUYBRECHTS, R., ET AL. 1998. Widespread occurrence of the micro-organism Wolbachia in ants. Proc. R. Soc. B, 265, 1447-1452.

WERren, J. H. 1997. Biology of Wolbachia. Ann. Rev. Entomol., 42, 587-609. 